less than $25 \%$ of his money on nutrients and the rest on palatability. His problem could be solved by methods outlined above for the dietitian.

We are grateful to Dr Watson for helpful suggestions in the preparation of this paper.

\title{
REFERENCES
}

British Medical Association (1938). Doctor's Cookery Book. London: British Medical Association. McCance, R. A. \& Widdowson, E. M. (1960). Spec. Rep. Ser. med. Res. Conn. no. 297.

Schultz, T. ( 1964). Human Needs Diet. Oxford University Institute of Economics and Statistics (mimeo.).

\section{Farm policies for the feeding of livestock}

\section{By S. C. Thompson, Department of Agriculture, University of Reading}

The animal feeds industry has known for some 25 years how to formulate a compound diet which conforms to a set of minimum or maximum nutrient constraints, and to ensure that the ingredients are chosen in such a way as to minimize the cost per ton of the resulting compound. The computer technique of linear programming has been in use for over Io years by the larger firms as a routine step in diet formulation, and it is accepted practice in England that minimum or maximum percentages of the more important nutrients are printed on the bag.

However, as Dent \& Casey ( 1967 ) remind us, animals do not eat percentages, but measurable weights of nutrients per day; and the total weight of the feed in which these nutrients are carried may be of secondary importance. Limits on voluntary intake will certainly impose extra constraints on the formulation of a ration but these will be by no means as rigid as the specification of fixed nutrient percentages. Formulation of a ration in terms of daily requirement for calories, amino acids, vitamins and minerals is no more complex a problem than that which faces the commercial compounder. $A$ linear programme can be used to devise both daily rations and percentage diets.

\section{Non-ruminant rations}

Dent (1964) took the question of ration formulation a step further by putting into an explicit form the notion that nutrients might substitute (in the economic sense) for one another while still producing a similar amount of end-product. Analysing results from growing pigs, he produced a series of functions relating daily live-weight gain, energy intake and crude protein intake. A stylised graph of one relationship is reproduced as Fig. I.

Two questions now present themselves to the stockman for simultaneous solution: what is the most desirable daily rate of gain; and for that gain, which particular combination of nutrients can be formulated most cheaply from the ingredients available? A least-cost ration for each of the three rates of gain drawn in Fig. I can 


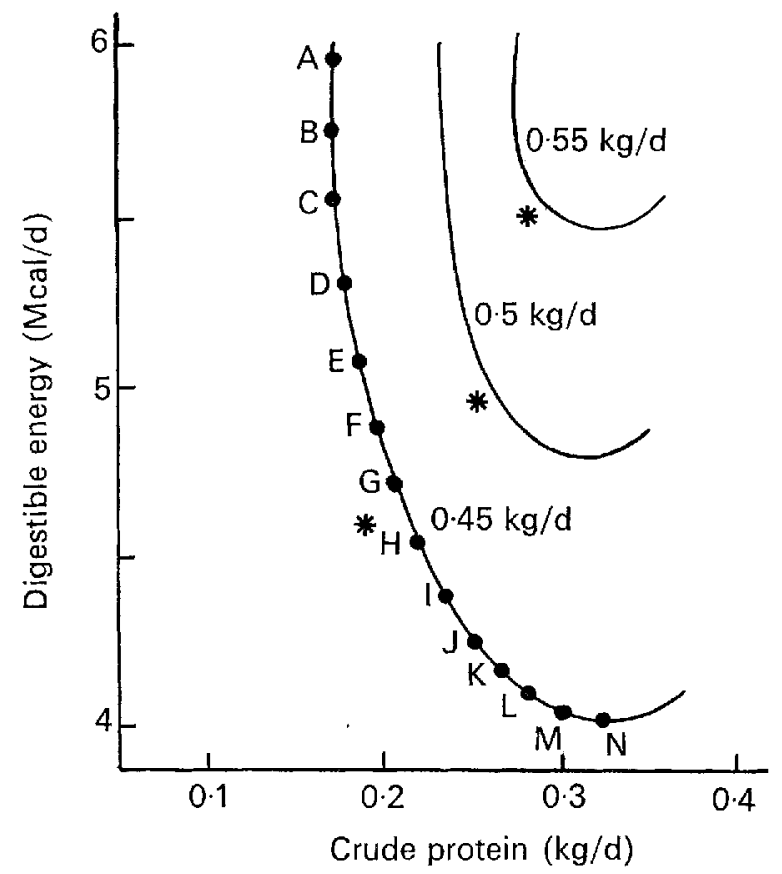

Fig. I. Isogrowth curves calculated for growing pigs fed varying combinations of crude protein and energy.

be found by solving a series of linear programmes. For the lowest rate of gain, for example, the nutrient specifications can be made to correspond with one of the points A to $\mathrm{N}$, and of the $\mathrm{N}$ 'least-cost' rations produced, one is the least cost of all, and is marked in the figure with an asterisk. Corresponding positions on the remaining growth isoquants are similarly marked.

Dent $\&$ Thompson $\left(19^{6} 7\right)$ have described an alternative method of computation, based on a Monte Carlo computer programme developed by Thompson (1967), which obviates the need for hand calculation. By means of random numbers the programme generates combinations of energy and protein at random, computes a ration to meet these specifications and links the cost per ton of the ration with the expected daily gain to produce a new criterion: cost $/ \mathrm{kg}$ gain. A thousand or more random rations can be computed in this way, and a graph is produced automatically to show the relationship between $\mathrm{kg}$ gain $/ \mathrm{d}$, and cost $/ \mathrm{kg}$ gain. Fig. 2 is an extract from a computer graph with these axes plotted for growing pigs.

Reference from the graph back to auxilliary computer print-out will supply the composition of the ration which corresponds to any particular point on the curve, but the choice now facing the farmer is whether to employ an expensive ration giving high growth rate or a cheap, slow ration. If the end-product of the growth process is saleable then it is a matter of calculus to determine the growth rate which maximizes annual sales over annual feed and other variable costs. The information required for such a calculation will include the sale price (adjusted for any quality differences arising from different rates of growth), costs of labour and veterinary 


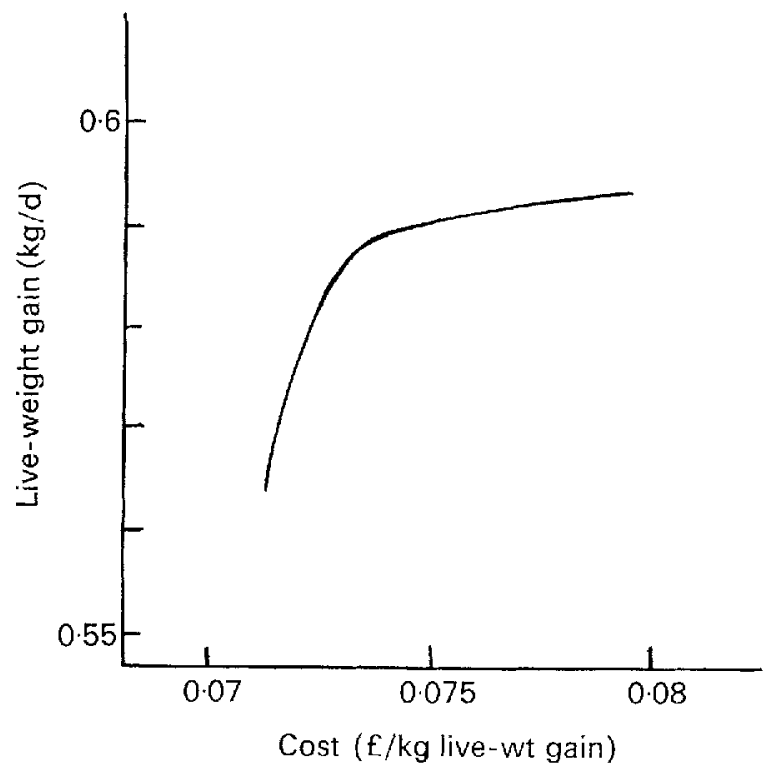

Fig. 2. Efficiency line relating daily live-weight gain in growing pigs to feed cost per kg live-weight gain, based on 1967 prices.

services, and the relationship described by Fig. 2. The computation involved in this final stage could usefully be inserted in a Monte Carlo programme to provide a package designed to maximize profit margin per pig space per year.

One complexity involved in practice is the need to split the production period for pigs into growing and fattening, where the nutrient requirements for a given rate of growth differ. A Monte Carlo programme, or a series of linear programmes for each growth period would be necessary, though the information required for calculation of the optimum growth rates in each period remains the same.

The nature of the environment has a substantial effect on the rate and quality of growth of all farm animals. The interaction of environment and level of feeding at the farm level is, however, an area where too little is known. Dent's growth functions are implicitly determined for one type of house, and the parameters of the equation would be expected to change under different conditions. Amount and type of capital investment will therefore be cxpected to influence feeding policy, and the concept of an overall optimum solution which embraces housing presents an enticing picture. Work along these lines is in progress at Reading University, where a Monte Carlo programme is being used to investigate the profitabilities and capital requirements of complete systems of pig production. Part of this work is described by Thompson (1969) with particular reference to weaner production, and lays stress on the need to view animal production at the farm level as an activity forming an integrated whole with the rest of the farm. A policy for feeding must in consequence be decided upon in the light of competition by quite different enterprises for common resources.

The shortcomings of linear programming and allied techniques when dealing $29(1) 9$ 
with rations for non-ruminants will be immediately apparent to specialists in nutrition. Not least the assumption of additivity of nutrients supplied by different ingredients, and the implication that no synergistic or depressive interactions occur between ingredients or nutrients. The influence of caloric density of the ration on intake and efficiency of conversion must therefore be ignored; although Johansson (1969), working with beef cattle in Sweden, proposes a search method involving a trial solution followed by a number of re-runs of the programme until an acceptable solution is found. Nevertheless, linear programmed rations, at least for hens and pigs, have been extensively tested in the field, and have been found to give satisfactory rates of production at a markedly lower cost than conventionally formulated rations. The testing of linear programmed rations, which in many cases contain rather unusual quantities and combinations of ingredients, has been instrumental in overcoming a number of taboos prevalent among stockmen.

\section{Ruminant rations}

Rations for ruminants present quite a different problem for the compounder in that he cannot control the amount of herbage taken in as a complement to his ration. Johansson (I969), in common with a number of other workers, has adopted a linear programming approach in which only hay and silage in controllable amounts are fed. The model is concerned with beef production; and a linear programme is incorporated as a subroutine in a larger programme which is concerned with calculating the animal's nutrient requirements at different stages of its life. Equations are developed for energy requirements for maintenance and production as functions of body-weight, required rate of gain, and caloric density of the ration. Protein, calcium and phosphorus requirements are similarly determined, and a limit on voluntary intake is formulated with regard to body-weight, total weight of feed, and percentage dry matter.

The model moves in steps through the production process, and at each stage stipulates a nutrient specification. The linear programme subroutine then devises a least-cost ration before the main programme moves on to the succeeding stage in the animal's life. The result is a series of rations, each optimum at a certain stage of growth.

Dean, Bath \& Olayide ( 1969 ) have extended the least-cost model for dairy cows by linking feed input with milk output to maximize money margin over feed costs. A set of maintenance and production constraints is formulated for each weight of cow and for its milking potential, and a separate programme is run for each set of constraints. They adopt the same strategy as Johansson in prohibiting the intake of fresh herbage, and prescribe a similar set of nutrient requirements. They treat the question of caloric density in a less sophisticated way than that proposed by Johansson, by dividing feeds into concentrates and roughage, and producing a tradeoff graph for the two types of ingredient. Fig. 3 represents their estimates for maximum voluntary intake by low-, medium- and high-yielding cows. These relationships are further simplified into two straight lines per curve for insertion into the linear programming matrix. Dean (1966) has developed tentative milk 


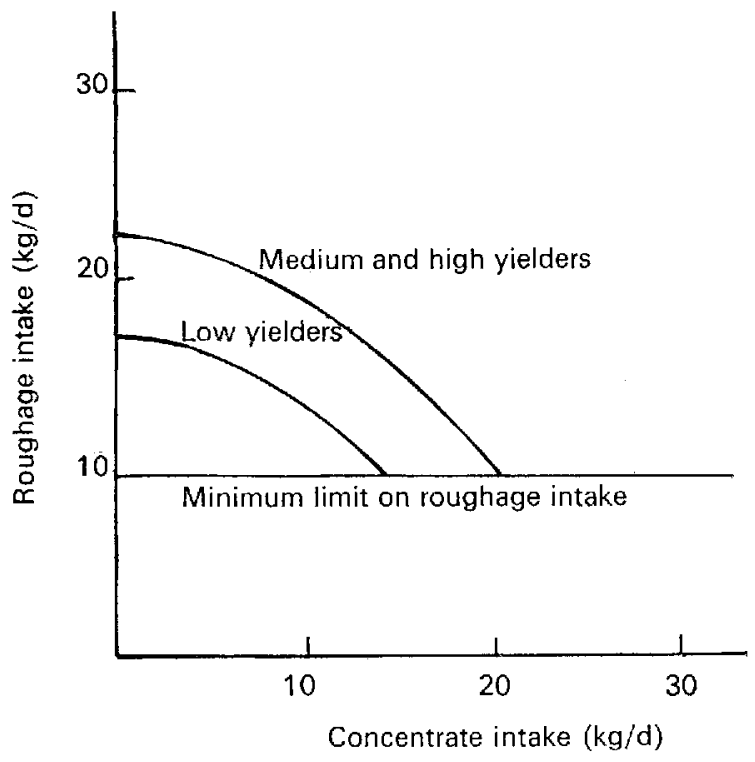

Fig. 3. Voluntary intake maxima for dairy cows fed different levels of concentrates.

production curves which show diminishing returns to estimated net energy intake, and Dean et al. ( $\left(\mathrm{I}^{6} 6 \mathrm{9}\right)$ have approximated the relationship by thirteen linear segments which have been incorporated into the linear model. Also incorporated in the model are minimum protein requirements for each level of milk production, and this serves to highlight one of the deficiencies of a purely linear programming approach. No interaction is allowed in the model between energy and protein, and milk production is limited by the nutrient in shortest supply, regardless of the level of the remaining nutrient which may possibly be in acute oversupply. An interaction between nutrients could be allowed for in a similar way to the function developed for pigs if a Monte Carlo or other simulation programme were used for the computation.

J. R. Crabtree (unpublished), working at the Grassland Research Institute is attempting a more ambitious model which, although it omits the milk output sector of production, does attempt to deal with the (zero) grazing animal and seasonal production of grass. Grass growth is described by a standard curve developed at the Institute. Zero grazing is assumed, which permits a controllable quantity of fresh material, to be included in the ration and any excess is conserved. A routine to take caloric density into account is included in the programme, and the result is a series of rations for succeeding periods of the year. The series is valid for a cow in a particular yield group, calving at a certain time of year and utilizing grassland managed under a fixed system. The logical extension of this model is to compute a series of rations for each cow, or group of cows in the herd, but the practicalities of feeding will influence the system chosen. Accepted practice at present is to feed cows an individual ration of concentrates while they are being milked, but time study data reveal that high yielders are already taking longer to eat their ration than to give milk. This, coupled with the investment needed and extra strain on the milker 
when he has other things to do, may lead to individual feeding outside the parlour, or to group feeding a complete ready-mixed ration $a d$ lib. The possibility of unrestricted feeding will lead to problems of choosing a common ration for a group of cows. Because of diminishing returns, the ration will be somewhat poorer than that required by the average of the group.

\section{Simulation methods}

The rigidities forced on the user by mathematical programming methods are judged by a number of workers to be intolerable when applied to ruminants, and especially to milk production. It is suggested that a process as complex as rumen fermentation cannot be modelled by a series of linear equations; and some alternative method must be found which, if necessary, abandons any aim of producing an optimum answer and concentrates instead on building a valid numerical model of the processes involved. This model can then be manipulated and extrapolated to a small degree beyond the observations which were used to build it.

Street ( 1969), working at Reading, and J. R. Crabtree (unpublished), have attempted models of this description for incorporation into larger systems embracing herd policy. The profusion and confusion of information at present available on the causal relationships involved in milk production appears to preclude the building of even a cursory model based on rumen processes, and in the construction of models to date most workers have contented themselves with mirroring accepted good practice at the farm level. A good deal of justification for this approach lies in the many buffers inherent in the animal-grazing relationship (Duckham 1967 ) which compensate for, and smooth out quite drastic changes in environment and feeding. The mobilization of body tissues to produce milk in the early part of lactation is an instance which throws doubt on the validity of using linear programming to compute a ration designed to attain an immediate rate of milk production. Preliminary results from models simulating herd performance indicate that feed costs, while amounting to some $50 \%$ of total costs, are not of paramount importance in explaining variation in herd profitability. Substantial variation, on the other hand, can be attributed to date of calving and calving interval.

The technique of model building for computers is as yet adolescent and most benefit accrues to the model builder himself. A further stage of development will be the use of these models in making and testing policy decisions, and as simulation becomes a more widely accepted technique it will play a greater role in complementing present optimizing techniques. Mybrid models which combine both aspects are beginning to emerge, and the dairy enterprise is one field in which they will be most effectively applied.

\section{REFERENCES}

Dean, G. W. (1966). Fifth Annual Dairy Cattle Day. University of California p. 18.

Dean, G. W.; Bath, D. L. \& Olayide, S. (1969). F. Dairy Sci. 52, 1008.

Dent, J. B. (1964). F. agric. Econ. 16, 68.

Dent, J. B. \& Casey, H. (1967). Linear Programming and Animal Nutrition. London: Crosby Lockwood $\&$ Son.

Dent, J. B. \& Thompson, S. C. (1967). Fm Economist II, 230. 
Duckham, A. N. (1967). Fodder Conservation Occasional Symposium no. 3, p. 1. British Grassland Society.

Johansson, V. (r969). Agricultural College of Sweden Department of Economics (mimeograph).

Street, P. R. (x 969$)$. Discussion paper for the Agricultural Economics Society, July r 969 (mimeograph).

Thompson, S. C. (1967). Study Reading University Department of Agriculture no. 3.

Thompson, S. C. (1969). Farm \& Country, September.

\section{Logging data in digital form}

By S. W. R. Cox, Instrumentation Department, National Institute of Agricultural Engineering, Silsoe, Bedfordshire

This paper is not written from any special knowledge of the data-logging requirements of nutrition experiments, but from some experience in assisting fellow scientists and engineers in logging and processing their 'data'. Powerful aid can be provided by digital computers, backed by data-logging equipment, but in individual cases the benefits have to be balanced against both the cost of automatic logging and the time required to programme a computer for the subsequent data processing Further deterrents may be unfamiliarity with the various forms of data recording equipment that are available commercially and uncertainty about their relative usefulness for a given experiment, for there is continuing advance in the performance of equipment and in the development of digital computers. In the latter there is a trend towards greater cost and power on the one hand, and towards smaller size, lower cost and increased portability on the other. Smaller computers, integral with data-logging units, may remove the convenient division between the logging and the processing stages of data handling.

Any survey of the cost or performance of data-logging techniques is liable to be out of date in a year or two, but certain types of equipment are now being produced commercially which will probably provide the basis of logging systems for some time to come. Statements about the spheres of application of such equipment are therefore of value.

Before attempting such a survey of data loggers the reasons for choosing digital equipment should be considered, for the stages of development at N.I.A.E. over the past few years (Matthews, 1967,1969 ) are probably relevant to aims of many other experimental establishments.

\section{Analogue measurements}

Until a few years ago most recorded measurements of N.I.A.E. were in analogue form on $\mathrm{Y}$ - $\mathrm{T}$ chart (i.e. with the $\mathrm{X}$-axis representing time, $\mathrm{T}$ ). The exceptions were normally those relating to counting systems where the output is normally in pulse or digital form. The single or multi-point chart recorder was the most widely used device. Its accuracy and speed of response suit many applications and, even in the multi-point form, with its discontinuous traces, it often provides a convenient visual presentation of the results, 